\title{
Disk Mass Densities in Edge-on Spiral Galaxies
}

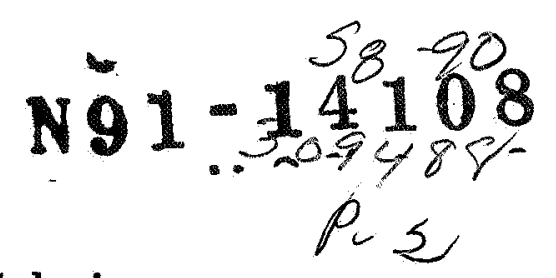

\author{
Michael P. Rupen
}

Center for Astrophysics

VLA observations of the neutral hydrogen gas in two nearby edge-on spirals (NGC 4565 and NGC 891) successfully resolve the thickness of the gas layers in both disks over a wide range in radii. The combination of ' $B$,' ' $C$,' and ' $D$ ' array data produces a $4 \operatorname{arcsec}(\sim 200 \mathrm{pc})$ beam and $21 \mathrm{~km} \mathrm{~s}^{-1}$ velocity resolution, combined with sensitivity to structures as large as 18 arcmin $(\sim 54 \mathrm{kpc})$. These observations directly constrain the mid-plane disk mass densities, under the assumption of an equilibrium between the thermal pressure of the gas and the gravitational attraction of the disk. The results of a preliminary analysis are as follows:

1. If the $z$-velocity dispersion of the gas is $10 \sigma_{10} \mathrm{~km} \mathrm{~s}^{-1}$, the mass densities in NGC 4565 fall from 0.11 to $0.009 \sigma_{10}^{2} M_{\odot} / \mathrm{pc}^{3}$ between 0.7 and 3.5 optical disk scale-lengths; the decline is exponential in radius, consistent with a constant mass-to-light ratio for the disk. In NGC 891, although the mass densities also fall off with radius (from 0.09 to $0.02 h \sigma_{10}^{2} M_{\odot} / \mathrm{pc}^{3}$ between 0.8 and 2.8 scale-lengths), there are abrupt local oscillations in the observed thickness that are difficult to model in terms of a simple hydrostatic equilibrium. Non-thermal pressures may account for these 'wiggles,' but the general trend of thickening with radius implies that the large-scale behavior of the thickness is determined by the balance of gravitation and thermal pressure.

2. If the disk mass distribution above the plane follows that of the old disk starlight, the mass-to-light ratio of the disk in NGC 4565 is $3_{-1}^{+3} \sigma_{10}^{2} M_{\odot} / L_{\odot, B}$, and the disk mass is about $15 \sigma_{10}^{2} \%$ of the total. The halo is probably dynamically important at all radii; the disk is not 'maximal,' unless the gas velocity dispersion is extremely high. If one assumes purely thermal pressures in NGC 891, its mass-to-light ratio increases with radius from $3 \pm 1$ to $5 \pm 2 h \sigma_{10}^{2} M_{\odot} / L_{\odot, B}$; again, the disk constitutes a small fraction of the mass.

3. In both galaxies, the atomic gas is an important dynamical component well before the edge of the disk, while the molecular gas may contribute a substantial fraction of the mid-plane mass density nearer the bulge.

These data also allow a detailed study of the HI in these galaxies; in general their brightness temperature distributions seem similar to that in the Milky Way. Both galaxies show asymmetric HI extensions beyond the optical disk. In NGC 4565 the extension is a surprisingly abrupt warp, which may bend back to parallel the galactic plane; the velocity structure implies the warp is continuous around the disk. 


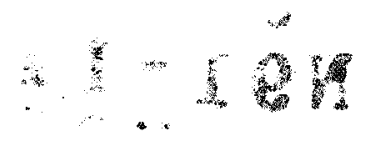

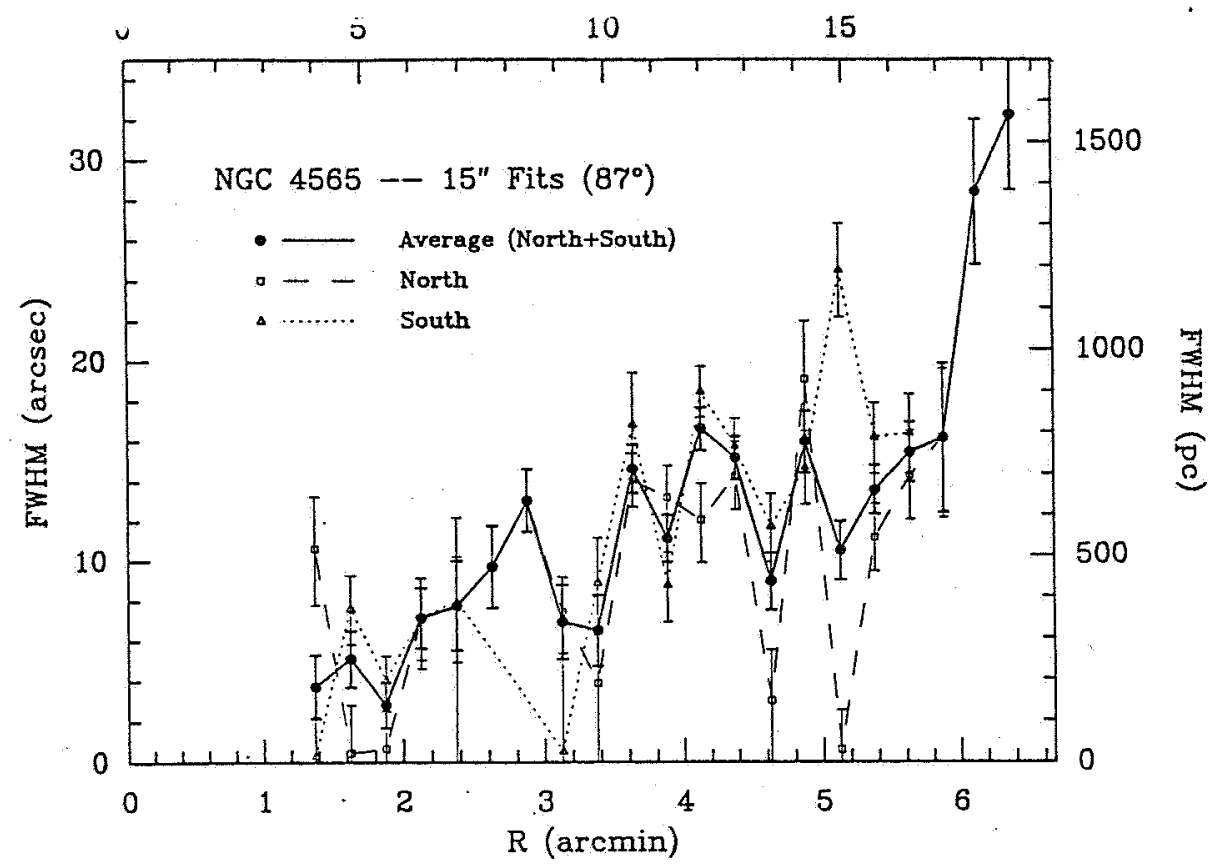

Fig. 1: Thickness of $\mathrm{HI}$ layer (FWHM). Inclination effects have been removed. Error bars are formal $1 \sigma$ errors of the fits. Note the consistency of the (independent) fits on opposite sides of the nucleus.

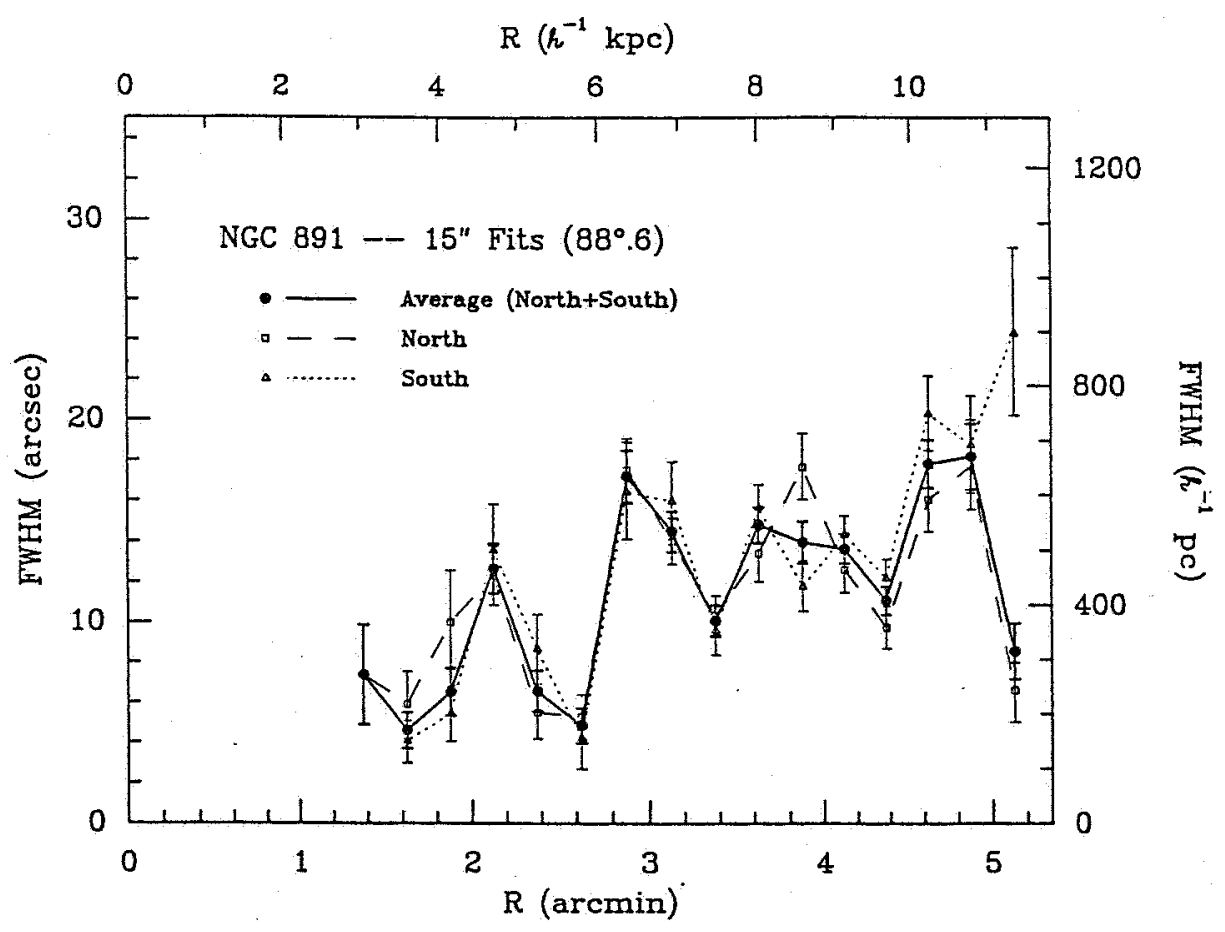

Fig. 2: Thickness of HI layer (FWHM). Inclination effects have been removed. Error bars are formal $1 \sigma$ errors of the fits. Note the abrupt "jumps" in the thicknesses. 